\title{
A Soft Museum Of Hardware Use \\ Testimonies from the Early Experience of Digital Devices as Historical, Pedagogical And Narrative Assets
}

\author{
Heitor Alvelos, University of Porto, Portugal \\ Daniel Brandão, University of Minho, Portugal \\ Abhishek Chatterjee, University of Porto, Portugal
}

The Asian Conference on Arts \& Humanities 2020

Official Conference Proceedings

\begin{abstract}
This research addresses the validation of narrative legacies of a first generation of digital and online media users upon its mass adoption in the 1980s and 1990s. As a complement to ongoing processes of technological obsolescence, whereby arcane digital media devices become potential museum objects or trending novelties, we vouch for the testimonies of early adopters: a transition from analogue to digitaldriven routines and competences was often symptomatic of semantic and subjective expectations, of cognitive, expressive, playful and mimetic processes. Often performed intuitively on relatively user-unfriendly hardware and software, early adoption of digital devices signalled a transition beyond the purely tangible or functional: it provided users with a felt need and desire for a paradigm shift that was yet to fully reveal itself, yet itself felt vaguely utopian. The paradigm of digital access and experience was still far from its current, seamless ubiquity - it often demanded personal effort and investment. However, this past experience is often regarded as an exercise in nostalgia, a mere path towards the ever-growing sophistication of current media devices; it is this tacit assumption that the current research questions, by bearing testimony to a singular historical moment of transition from analogue to digital environments - with all the challenges this entailed. The ongoing research is performed via semi-structured, recorded interviews with early adopters. The interviews are recorded, and the contained narration provides the primary source material for extrapolation, pattern recognition and storytelling. The outcomes are intended to serve historical, broadcasting, pedagogical and philosophical contexts.
\end{abstract}

Keywords: Digital Transition, Design Research, Heritage Preservation, Digital Storytelling

\section{iafor}

The International Academic Forum

www.iafor.org 


\section{Introduction}

This paper addresses the validation of narrative legacies of the starting generation of digital and online media users upon its mass adoption in the 1980s and 1990s. The study is adjunct to a design research and mediation project, 'Anti-Amnesia', that focuses on the recovery and restoration of knowledge embedded in traditional industrial practices that are currently on the brink of dissipation. The project is based in the Research Institute for Design, Media and Culture's (ID+) Unexpected Media Lab (LUME), and situated at the Faculty of Fine Arts, University of Porto.

A critical component of Anti-Amnesia's ongoing investigation is devoted to the auscultation of a plethora of challenges faced by traditional makers due to the hastened onfall of technological obsolescence brought about by rapidly evolving production, consumption, and distribution standards. The research accordingly questions the imperativeness of digital literacy in cultural ecosystems that have historically maintained a different interpretation of progress. It works closely, in this regard, with individuals from traditional industrial cultures and craft communities to understand and situate the dynamics of the occurring conflict between convention and contemporaneity: with a view to develop generative resources such as product and communication strategies, intergenerational and interdisciplinary knowledge networks, and mediation models based on active pedagogy. An institutive reasoning behind these measures is to abut the revivification of traditional praxis as scaffold, but with a requisite amount of sensitivity and discretion so as not to compromise the associated cultural identities and creative/processual legacies.

The leitmotif that connects Anti-Amnesia's strategic objectives with the study in question pertains to the qualitative multiplicity of the term 'media', wherein its understanding as a medium of expression in manual crafts finds consonance with theoretical and practical paradigms of the digital domain, such as in terms of formats, applications, channels, and gadgetry. The study therefore intends to draw attention to the risk of loss which media heritage (both digital and cultural) is exposed to as a consequence of the accelerated obsolescence of the underpinning tools, processes, and mechanisms, alongside some other depreciative factors such as resource uncertainties and public and administrative apathy towards past standards (Lusenet \& Wintermans, 2007).

Thus, as a complement to ongoing processes of technological obsolescence, whereby arcane digital media devices become potential museum objects or trending novelties, the study vouches for the testimonies of early adopters: a transition from analogue to digital-driven routines and competences was often symptomatic of semantic and subjective expectations, of cognitive, expressive, playful and mimetic processes. In agreement with Baetens (2017), the corollary is not to generate a "different" perspective, but rather to acknowledge oral histories that may not find a place in conventional forms of discourse on cultural heritage. In this regard, Schleser (2012) points out that the process of documenting these events forms the basis for constructing cultural memory, and that such "unintentional archives" are "unique ecologies", presenting accurate and unfiltered reflections on everyday life, that can help foster and engage future critical interdisciplinary work. 
Lusenet \& Wintermans (2007) consider digital media objects as embodying certain peculiarities that differentiate them from traditional heritage materials: "they know no boundaries, they are dynamic, interactive and fragmented." Spigel (2010), in conjunction, views old hardware as "evocative objects, whose material presence reemerges in scholarly discussions, and in archiving practices, with the emergence of digital means", as can be evidenced forthwith from this particular discourse. Yet besides this material presence, the memory of both analogue engagement and an intuitive tap into a felt inevitability are themselves worthy of analysis: among the determinism of technological progress, the project argues that we have often neglected to observe the competences and dynamics left behind as a consequence. It is precisely these dynamics of transition that the research proposes to preserve, enquire, and vouch for.

The study's methodological approach correspondingly finds alignment with Bastian and Harvey's (2012) perplexities regarding approach, wherein, objectives driven by a pedagogical interest in identifying and experimenting with a theoretical infrastructure, that can accommodate the explication of digital transition, require to be articulated with the need to better understand the dichotomy arising between the perceptible allowances of contemporary technology towards heritage preservation, and its antithetical role in the abetment of dictated obsolescence.

\section{Testimonials from Early Adopters}

Often performed intuitively on relatively user-unfriendly hardware and software, early adoption of digital devices signalled a transition beyond the purely tangible or functional: it provided users with a felt need and desire for a paradigm shift that was yet to fully reveal itself, yet itself felt vaguely utopian. This paradigm of digital access and experience was still far from its current, seamless ubiquity - it often demanded personal effort and investment. As pointed out by Smith (2016), “...many folks weren't ready, or maybe they didn't care enough about their TV signal quality to upgrade."

The project's argument in aggregating these mostly personal memories of early digital use is that cumulatively they may reveal patterns and taxonomies, and present themselves as heritage: since the default expectation towards technology was not that it would be seamless, as a first approach to a digital device was often mimetic of prior, analogue procedures, and digital conversion offered the illusion of perpetuity. It is not about arguing that "those were the days", but rather about building an empirical sensitivity to what changes in ourselves through technological progress, so that we may cultivate a clearer vision of this change beyond the benevolent but tacit assumptions around innovation and progress - with inevitable impact on the ways we appreciate and incorporate the aforementioned, traditional crafts and manufactures.

Reflections on past experience can, however, often be reductively regarded as an exercise in nostalgia, a mere segment of a trajectory that leads towards the evergrowing sophistication of current media devices. It is this tacit assumption that the current study questions, by bearing testimony to a singular historical moment of transition from analogue to digital environments - with all the challenges this entailed. Recognising such ambiguity surrounding nostalgia, Bevan (2013) states that invocations to the past and the associated material connections to a particular period 
may not be rooted in material realities of that time, and that it "reinforces the contradiction inherent to nostalgia as a yearning for the past, knowledge of the impossibility of return, and simultaneous understanding that the idyllic past was never real to begin with.".

\section{Premises and expectations}

The study is being performed via semi-structured, recorded interviews with early adopters, whose contained narration provides the primary source material for extrapolation, pattern recognition and storytelling. The outcomes are intended to serve historical, broadcasting, pedagogical and philosophical contexts. The consequent insights are intended to address a series of critical questions pertaining to the digital, such as:

- the various impresses of the transition from analogue to digital on individual facticities and collective realities;

- $\quad$ the proliferation of virtual constructs as a new limit of authenticity;

- the emergence of virtual sentiment lexicons as proxies for emotional communication (Santamaría-Bonfil \& López, 2020);

- the influences of uncritical consumption (Gauntlett, 2002) on cultural interpellation.

These theoretical inquisitions, among others to be added cumulatively, are expected to derive critical reflections on the interlacing between change and continuity, alongside shedding light on the "implications of an unprecedented accumulation of media and cultural resources, and their potential for ways of making sense of our own and others' experience over time" (Keightley \& Schlesinger, 2014). In concordance, Schleser (2012) sees merit in reading the generated information as temporal evidence, stating the imperativeness of embracing such wealth of "born-digital cultural production located within unintentional archival spaces... that are located outside the conventional domain of traditional cultural heritage institutions", in order for future generations to accurately comprehend past realities.

The nature of inquiry is conversational, wherein nostalgia is used tactically to expose sets of "internal contradictions" within the digital, and to "serve as a space for a mnemonic processing of cultural memories of old media, as popular conjecture on the present trajectory of media technology" (Bevan, 2013). The interviewees essentially represent participant-observers in this broad transformation, recording changes as they occur while contributing to new waves of change. Their accounts stand as testament to the overriding nature of digital upheaval, wherein generations of technologies and software are introduced on a recurring basis with the compulsion to further transform markets, society, and everyday life (Hofacker, 2019).

The current geo-political predicament we are traversing may somewhat tempt us into this reading that before the ubiquity of smart technology, geo-tagging, social media, hacking, viruses and bot farms, things were indeed better, but rather than amplify that speculation, we posit that it is worth looking into the last era where the digital was not taken for granted. The issue is also lexical: 'memory' as data storage is also 'memory' as a wealth of personal experience (Niemeyer, 2019) - and ultimately signals a collective convergence into a zeitgeist that may be best recognised retroactively in order to actively inform the present. 
The research is being developed within the framework of the project "Anti-Amnesia: design research as an agent for narrative and material regeneration and reinvention of vanishing Portuguese manufacturing cultures and techniques", co-financed by Portugal 2020, alongside the European Regional Development Fund and the Foundation for Science and Technology, Portugal (ID+/Unexpected Media Lab: POCI-01-0145-FEDER-029022; 2018-2020).

More information about the study and how to participate can be found at: http://softmuseumhardwareuse.wordpress.com

Information about Project Anti-Amnesia can be found at: http://endlessend.up.pt/antiamnesia 


\section{References}

Baetens, J. (2017). Rogue Archives: Digital Cultural Memory and Media FandomRogue Archives: Digital Cultural Memory and Media Fandom by Abigail De Kosnik. MIT Press, Cambridge, MA, U.S.A., 2016. Leonardo, 50(5), 538-539. doi: 10.1162/leon_r_01502

Bastian, J.A \& Harvey, R. (2012). The convergence of cultural heritage, Proceedings of The Memory of the World in the Digital Age: Digitization and Preservation - An international conference on permanent access to digital documentary heritage, 650659. Retrieved from:

http://www.unesco.org/new/fileadmin/MULTIMEDIA/HQ/CI/CI/pdf/mow/mow_van couver_programme_en.pdf

Bevan, A. (2013). Archiving the Digital Transition in the Boomer TV Sitcom Remake. Adaptation, 6(3), 305-319. doi: 10.1093/adaptation/apt007

Gauntlett, D. (2002). Media, Gender and Identity. London: Routledge.

Hofacker, Charles. (2019). The Growing Importance of Software as a Driver of Value Exchange. doi: 10.1108/S1548-643520190000016007.

Keightley, E., \& Schlesinger, P. (2014) Digital media - social memory: remembering in digitally networked times. Media, Culture \& Society, 36 (6). 745-747. ISSN: $0163-$ 4437

Lusenet, Y. de, \& Wintermans, V. (2007). Preserving the digital heritage: principles and policies: selected papers of the international conference organized by Netherlands National Commission for Unesco, Koninklijke Bibliotheek (National library of the Netherlands): The Hague, 4-5 November 2005. Amsterdam: Netherlands National Commission for UNESCO

Niemeyer, K. (2019). Digital Memory Studies: Media Pasts in Transition. Historical Journal of Film, Radio and Television, 39(1), 189-191. doi:

10.1080/01439685.2018.1518196

Santamaría-Bonfil, G., \& López, O. G. (2020). Emoji as a Proxy of Emotional Communication. Becoming Human with Humanoid - From Physical Interaction to Social Intelligence. doi:10.5772/intechopen. 88636

Schleser, J. (2012). Unprotected Memory, Proceedings of The Memory of the World in the Digital Age: Digitization and Preservation - An international conference on permanent access to digital documentary heritage, 378-392. Retrieved from: http://www.unesco.org/new/fileadmin/MULTIMEDIA/HQ/CI/CI/pdf/mow/mow_van couver_programme_en.pdf

Smith, E. (2016). The Long, Weird Transition from Analog to Digital Television. Retrieved from: https://www.atlasobscura.com/articles/the-long-weird-transitionfrom-analog-to-digital-television 
Spigel, L. (2010) "Housing Television: Architectures of the Archive." The Communication Review, 13(1), 52-74.

Contact Email: halvelos@fba.up.pt 